Diabetologia 10, 97-100 (1974)

(C) by Springer-Verlag 1974

\title{
Intralipid Triglyceride and Oral Glucose Tolerance
}

\author{
T. Gibson, J.H. Fuller, S.L. Grainger, R.J. Jarrett and H. Keen \\ Unit for Metabolic Medicine, Department of Medicine, Guy's Hospital, London, SE 1 9RT, Great Britain
}

Received: June 27, 1973, and in revised form: October 29, 1973

\begin{abstract}
Summary. The inter-relation between hypertriglyceridaemia and glucose intolerance has been studied experimentally in fifteen male subjects by measuring the effect of acute elevation of plasma triglyceride (injection of Intralipid intravenously) upon oral glucose tolerance. The induced hypertriglyceridaemia did not alter the mean blood glucose or plasma insulin response to oral glucose.
\end{abstract}

The associations between plasma triglyceride levels and glucose tolerance observed both in general population studies and in "patient" populations are probably due to a mutual link with a further factor or factors.

Key words: Triglycerides, fat tolerance test, oral glucose tolerance, plasma insulin.
There is a complex relationship between glucose and insulin metabolism on the one hand and triglyceride metabolism on the other. Plasma triglyceride levels are commonly raised in diabetes [5] and there is a high frequency of glucose intolerance in individuals found with elevated levels of very low density lipo. protein ("prebeta" lipoprotein) [11]. In an earlier study of a sample of a normal working population $[6,1]$ we found that the fasting plasma triglyceride level correlated very strongly with the blood sugar response in an oral glucose tolerance test; its correlation with plasma insulin response was of a lower order. The simplest explanation of this relationship between triglyceride levels and glucose tolerance, suggested by Albrink and Davidson (1966), is that circulating triglyceride in some way directly affects the metabolism of the glucose load. The present investigation was planned to test this hypothesis.

\section{Material and Methods}

The subjects studied were 15 male hospital in-patients with minor conditions not overtly affecting metabolism. Their mean age was $44 \pm 4.34$ S.E.M. years and mean weight $70.3 \pm 2.96 \mathrm{~kg}$. They received a full hospital in-patient diet containing at least $250 \mathrm{~g}$ carbohydrate daily. The following procedures were performed in random order in the morning after an overnight fast of $14 \mathrm{~h}$ :

1. A standard two-hour $50 \mathrm{~g}$ oral glucose tolerance test (OGTT).

2. An identical two-hour $50 \mathrm{~g}$ oral glucose tolerance test with $10 \%$ Intralipid (special experimental batch No. 199523). $1 \mathrm{ml} / \mathrm{kg}$ body weight was administered intravenously over two minutes as the glucose load was drunk (ILOGTT).

3. Intravenous Intralipid $(10 \%), 1 \mathrm{ml} / \mathrm{kg}$ body weight, administered by itself over two minutes (IL).

Venous blood samples were obtained from an indwelling catheter in an antecubital vein before and at intervals of five minutes after the glucose and/or Intralipid load for one hour, thereafter at 90 and $120 \mathrm{~min}$. The three tests were performed at intervals of four to seven days. Blood sugar was measured by the ferricyanide reduction micro method (Technicon method N-9a) on an Auto Analyser, and plasma immunoreactive insulin by a double antibody method using the Amersham kit. The intravenous lipid tolerance test is that described by Lewis et al. (1972) in which plasma Intralipid triglyceride is measured by nephelometry, using plasma diluted to $1 / 50$ with $0.9 \%$ saline.

\section{Results}

The mean blood sugar values in the three tests are shown in Table 1 . There is a very small difference in the ascending limb of the glucose response with and without Intralipid. However, the mean fasting blood sugar levels differed slightly on the two occasions, so the results have been expressed as percentage changes from fasting levels (see Fig. 1). Treated in this way the differences between the two blood sugar curves are insignificant. There was no significant effect of Intralipid upon fasting blood sugar levels (Table 1).

Mean insulin levels in the three tests are shown in Table 2. The insulin responses to glucose, with and without Intralipid, are almost superposable. There was equally little effect of glucose ingestion on Intralipid disposal. The calculated half time of disappearance of the Intralipid glyceride was $16.9 \pm 1.2 \mathrm{~min}$ when glucose was given and $16.2 \pm 1.9 \mathrm{~min}$ when it was not (Fig. 2).

\section{Discussion}

There is clearly remarkably little direct effect of a large rise of circulating triglyceride upon oral glucose tolerance or of oral glucose upon the triglyceride re- 
moval rate. The lack of effect upon blood sugar levels is in apparent conflict with the findings of Felber and Vannotti (1964). In their study, however, the Intralipid. was administered by infusion for two hours before the glucose load and continued during the three hours of the test. The lipid infusion was accompanied by elevation of free fatty acid (FFA) levels and this, rather than the hypertriglyceridaemia, may have been responsible for the observed impairment of glucose tolerance. In our study, any rise in FFA levels would presumably have been too late to affect the disposal of the ingested glucose.

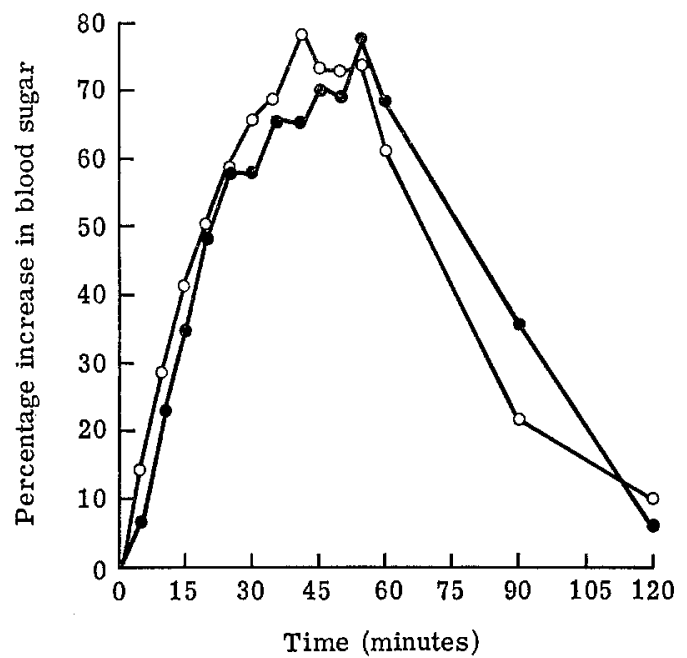

Fig. 1. Mean blood sugar changes in the two OGTT expressed as a percentage of the fasting level of blood sugar. Closed symbols $=$ OGTT, open symbols $=$ OGTT + Intralipid

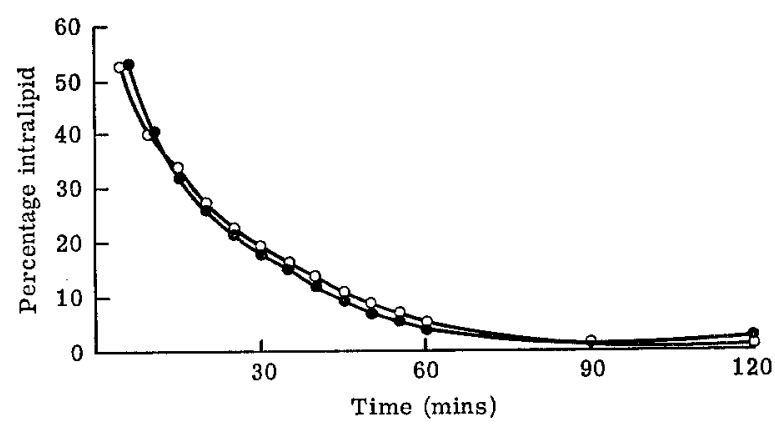

a. with $50 \mathrm{~g}$ oral glucose $\multimap\left(\mathrm{T}^{\frac{1}{2}}=16.9 \mathrm{~min}\right)$

b. with intralipid alone $\longrightarrow\left(\mathrm{T}^{\frac{1}{2}}=16.2 \mathrm{~min}\right)$

Fig. 2. Mean disappearance curves for Intralipid in the two OGT'T

The absence of an effect of intravenous triglyceride upon fasting plasma insulin levels is in accord with the results of other investigations $[9,8]$, although several workers have reported an augmentation of the insulin response to oral or intravenous glucose administered during a prolonged infusion of triglyceride $[9,16]$. Nikkilä and Taskinen (1970) demonstrated a prompt,

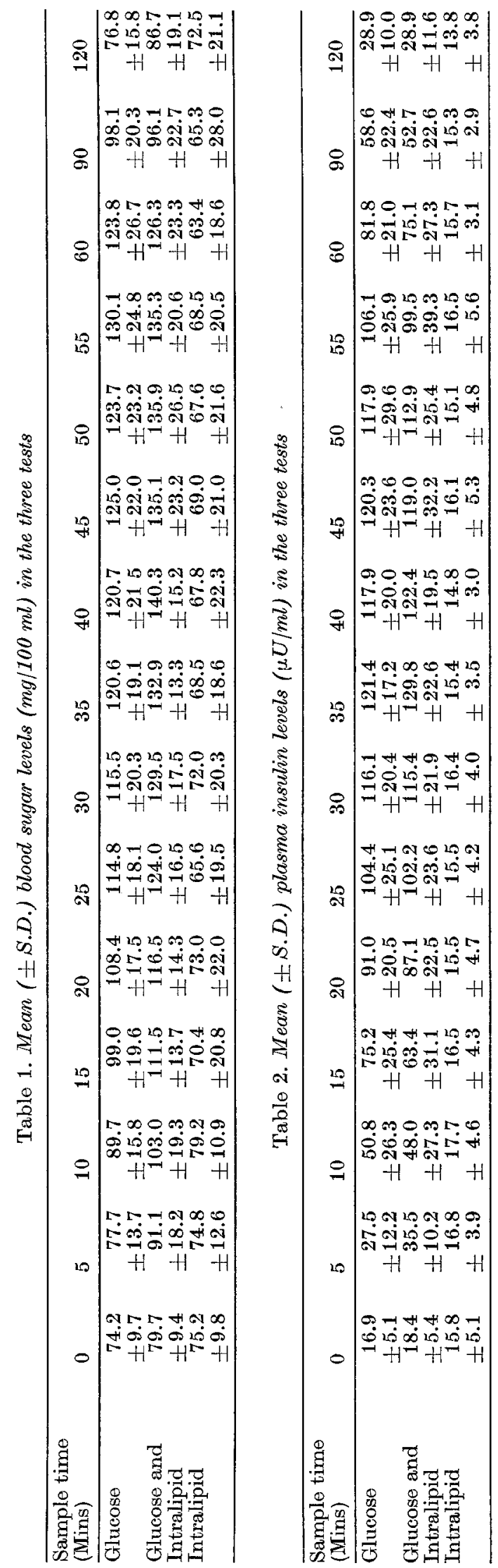


but small, increase of plasma insulin at the start of an Intralipid infusion into subjects already being infused with glucose. In the first situation [9] the probable effects of the raised FFA level must be considered in the explanation. In the second situation [14], the infusion of Intralipid was started when insulin levels were already elevated by the glucose infusion.

The explanation of the observed link between hypertriglyceridaemia and glucose intolerance would seem, on the basis of our findings, unlikely to be due to a simple, direct effect of circulating triglyceride upon the disposal of glucose. However, it might be argued that the experimentally induced hypertriglyceridaemia (giving a mean peak plasma concentration of $135 \mathrm{mg} /$ $100 \mathrm{ml}$ above the fasting level) was in some way inadequate to achieve such an effect. For example, the raised level of endogenous plasma triglyceride associated with glucose intolerance is usually in the form of very low density lipoprotein particles, whereas Intralipid is in the form of the larger, chylomicron-like particles. Glucose intolerance is not a feature of the uncommon hyperlipidaemia characterised by hyperchylomicronaemia ("clearing factor deficiency", "Type 1" of Frederickson et al. (1967)). Furthermore, a peak of hypertriglyceridaemia, provoked by intravenous bolus injection, might not exert the same metabolic effects on carbohydrate metabolism as exposure to longer-lasting though lower levels. Unfortunately, long infusions of Intralipid introduce the confounding factor of a progressively rising concentration of FFA, which itself will provoke glucose intolerance [15].

Suggestive though less direct evidence that glucose intolerance is not a direct effect of hypertriglyceridaemia may be adduced from other studies. Bagdade et al. (1971) reported that clofibrate lowered endogenous triglyceride levels by almost $50 \%$, but had no effect on basal glucose or insulin concentrations. Further, the same group showed that the aggravation of hyperlipidaemia with high dietary carbohydrate intake ( $85 \%$ of calories) was accompanied not by deterioration but by significant improvement in oral glucose tolerance [7]. Similarly, Owen et al. (1971) induced varying rises of plasma triglyceride levels with an $85 \%$ carbohydrate diet and were unable to show any correlated change in glucose tolerance, though changes in insulin levels showed some relationship. The Seattle group are more inclined to attribute the glucose intolerance and hyperinsulinaemia of hypertriglyceridaemia to the frequently associated obesity, a conclusion endorsed by Berchtold, Bjorntorp et al. (1972) from direct study of fat cell size in men after myocardial infarction. However, the findings of Wilson et al. (1971) suggest that reduction of plasma lipids by increased polyunsaturated dietary fatty acids is accompanied by improvement in glucose tolerance; despite unrestricted carbohydrate intake and a mean weight loss of less than $1 \%$.

The weight of evidence at present therefore suggests that although glucose tolerance and glyceridae- mia are often closely associated the link is not one of cause and effect but that both are the linked consequence of some independent causal factor or factors.

Acknowledgements. We acknowledge with gratitude the technical assistance in this study of Mr. Alan Schwartz, Miss Yolande Roth and Mr. Michael Wood.

All the subjects were fully informed volunteers and we are very grateful for their co-operation.

The Intralipid used was kindly supplied by Messrs. Paines and Byrne on behalf of Vitrum, Stockholm.

J.H. Fuller is supported by a grant from the Medical Research Council.

\section{References}

1. Abrams, M.E., Jarrett, R.J., Keen, H., Boyns, D.R., Crossley, J.N.: Oral glucose tolerance and related factors in a normal population sample. II. Inter-relationship of glycerides, cholesterol and other factors with the glucose and insulin response. Brit. med. J. 1969 I, 599 -602

2. Albrink, M.J., Davidson, P.C.: Impaired glucose tolerance in patients with hypertriglyceridaemia. J. Lab. clin. Med. 67, 573-584 (1966)

3. Bagdade, J.D., Bierman, E.L., Porte, D.: Influence of obesity on the relationship between insulin and triglyceride levels in endogenous hypertriglyceridemia. Diabetes 20, 664-672 (1971)

4. Berchtold, P., Björntorp, P., Gustafson, A., Lindholm, B., Tibblin, G., Wilhelmsen, L.: Glucose tolerance, plasma insulin and lipids in relation to adipose tissue cellularity in men after myocardial infarction. Acta. med. scand. 191, 35-41 (1972)

5. Bierman, E.L., Porte, D.: Carbohydrate intolerance and lipemia. Ann. Intern. Med. 68, 926-932 (1968)

6. Boyns, D.R., Crossley, J.N., Abrams, M.E., Jarrett, R.J., Keen, H.: Oral glucose tolerance and related factors in a normal population sample. I. Blood sugar, plasma insulin, glyceride and cholesterol measurements and the effects of age and sex. Brit. med. J. 1969 I, 595- 598

7. Brunzell, J.D., Lerner, R.L., Hazzard, W.R., Porte, D., Bierman, E.I.: Improved glucose tolerance with high carbohydrate feeding in mild diabetes. New Engl. J. Med. 284, 521-524 (1971)

8. Carroll, K.F., Nestel, P.J.: Effect of long-chain triglyceride on human insulin secretion. Diabetes 21, 923-929 (1972)

9. Felber, J.F., Vannotti, A.: Effects of fat infusion on glucose tolerance and insulin plasma levels. Med. Exp. 10, $153-156$ (1964)

10. Frederickson, D.S., Levy, R.I., Lees, R.S.: Fat transport in lipoproteins - an integrated approach to mechanisms and disorders. New Engl. J. Med. 276, $32-44$ et seq. (1967)

11. Glueck, C.J., Levy, R.I., Frederickson, D.S.: Immuno-reactive insulin, glucose tolerance and carbohydrate inducibility in Types II, III, IV and V hyperlipoproteinemia. Diabetes 18, 739-747 (1969)

12. Lewis, B., Böberg, J., Mancini, M., Carlson, L.A.: Determination of the intravenous fat tolerance test with Intralipid by nephelometry. Atheroselerosis 15, $83 \rightarrow 86(1972)$

13. Owen, W.C., Kreisberg, R.A., Siegal, A.M.: Carbohydrate-induced hypertriglyceridemia: inhibition by phenformin. Diabetes 20, $739-744$ (1971)

14. Nikkilä, E.A., Taskinen, M.R.: Hypertriglyceridemia and insulin secretion, p. 220. In: "Atherosclerosis", Proceedings of the Second International Symposium, (Ed. Jones, R.J.). New York: Springer-Verlag 1970 
15. Seyffert, W.A., Jr., Madison, L. L. : Physiologic effects of metabolic fuels on carbohydrate metabolism. I. Acute effect of elevation of plasma free fatty acids on hepatic glucose output, peripheral glucose utilisation, serum insulin and plasma, glucagon levels. Diabetes 16, $765-776(1967)$

16. Thorell, J., Persson, B., Sterky, E.: Effect of fat infusion on plasma glucose, FFA, glycerol and insulin levels during intravenous and oral glucose tolerance tests (Abstract). Diabetologia 2, 232 (1966).
17. Wilson, W.S., Hulley, S.B., Burrows, M.I.: Serial lipid and lipoprotein responses to the A.H.A. fat-controlled diet. Amer. J. Med. 51, $491-503$ (1971)

Dr. R.J. Jarrett

Dept. of Medicine

Guy's Hospital Med. School

London SE1 $9 \mathrm{RT}$

Great Britain 